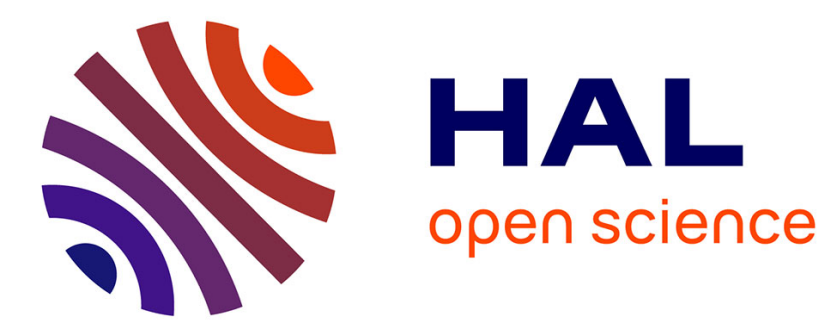

\title{
Un (anti)racisme légitime dans les médias ?
}

Marion Dalibert

\section{To cite this version:}

Marion Dalibert. Un (anti)racisme légitime dans les médias ?. Mouvements: des idées et des luttes, 2014, 79, pp.139-147. 10.3917/mouv.079.0139 . hal-01612657

\section{HAL Id: hal-01612657 https://hal.science/hal-01612657}

Submitted on 10 Oct 2017

HAL is a multi-disciplinary open access archive for the deposit and dissemination of scientific research documents, whether they are published or not. The documents may come from teaching and research institutions in France or abroad, or from public or private research centers.
L'archive ouverte pluridisciplinaire HAL, est destinée au dépôt et à la diffusion de documents scientifiques de niveau recherche, publiés ou non, émanant des établissements d'enseignement et de recherche français ou étrangers, des laboratoires publics ou privés. 
Marion Dalibert

Laboratoire GERiiCO - Université Lille 3

\section{Un (anti)racisme légitime dans les médias?}

Le mouvement des Indigènes de la République (MIR) est un collectif protestataire dont l'existence publique a débuté en janvier 2005 suite à la diffusion de l'appel « Nous sommes les Indigènes de la République ! », pétition qui dénonce les discriminations raciales causées, selon le collectif, par l'idéologie coloniale qui perdure dans la société française. En faisant l'analogie entre la position sociale des minorités ethnoraciales avec celle des indigènes des colonies, et en demandant à ce que "la France » ou "l'État et la société » opèrent «un retour critique radical sur leur passé-présent colonial ${ }^{1} »$, les initiateurs de l'appel ont mis en cause la participation de la nation, c'est-à-dire l'État et ses membres, dans la manifestation des rapports de pouvoir liés à la race ${ }^{2}$. Le mouvement a donc dénoncé la (re)production du racisme systémique et institutionnel, racisme dont la responsabilité est collective et non pas individuelle.

Ce discours, relativement inédit en France, a eu des effets sur la couverture médiatique $\mathrm{du}$ MIR qui interroge le fonctionnement de la sphère publique française quant aux revendications antiracistes qui peuvent y être portées. Au regard des mouvements sociaux qui ont eu une existence publique ces trente dernières années, on remarque que ceux qui sont passés aux agendas médiatique et politique ont dénoncé l'existence de discriminations directes, c'est-à-dire visibles et/ou entendables (comme les agressions et propos racistes), et qui ont désigné comme coupable (et comme adversaire) un «Autre» explicitement discriminant et disqualifié socialement, à l'instar de la Marche pour l'égalité et contre le racisme de 1983 qui s'est mobilisée en réaction à la montée du Front national et à l'augmentation des crimes contre les personnes issues de l'immigration et, à sa suite, SOSRacisme. L'étude de la médiatisation du MIR et de la réception de l'appel dans les journaux télévisés (JT) de TF1, France 2 et France 3 et au sein des articles du Figaro, du Monde et de Libération de janvier 2005 à décembre 2010 (ce qui fait, en totalité, 42 articles et 2 sujets de $\mathrm{JT}$ ) révèle que le mouvement a été faiblement et négativement médiatisé, et que la thèse du continuum colonial portée par le MIR, et qui a constitué le cadrage principal des articles, a été débattue et, au final, contre-argumentée.

\section{La médiatisation du mouvement comme déni de reconnaissance sociale}

Après avoir fait circuler l'appel « Nous sommes les Indigènes de la République ! » par courriel en janvier 2005, les membres du MIR l'ont adressé à certaines rédactions, notamment à la presse quotidienne nationale dite « de référence » (le Figaro, le Monde, Libération), en espérant qu'elles le publient ou que certains journalistes rendent compte de leur mobilisation. Or, celui-ci n'a jamais fait l'objet d'une tribune libre et le MIR a été quasiment invisible à la télévision (graphique 1) — seul TF1 lui a consacré une brève en images en mai 2005 et 2006.

Graphique 1 - Nombre d'articles de presse et de sujets de JT publiés/diffusés sur le MIR par

\footnotetext{
Se référer à l'appel du MIR. Initiateurs-trices (2005), « Nous sommes les Indigènes de la République » in Les Mots sont importants.

Nous utilisons la notion anglo-saxonne de race. La race n'a évidemment pas d'existence biologique, mais certaines caractéristiques physiques sont des signifiants, construits socialement comme tels, qui induisent son existence sociale.
} 


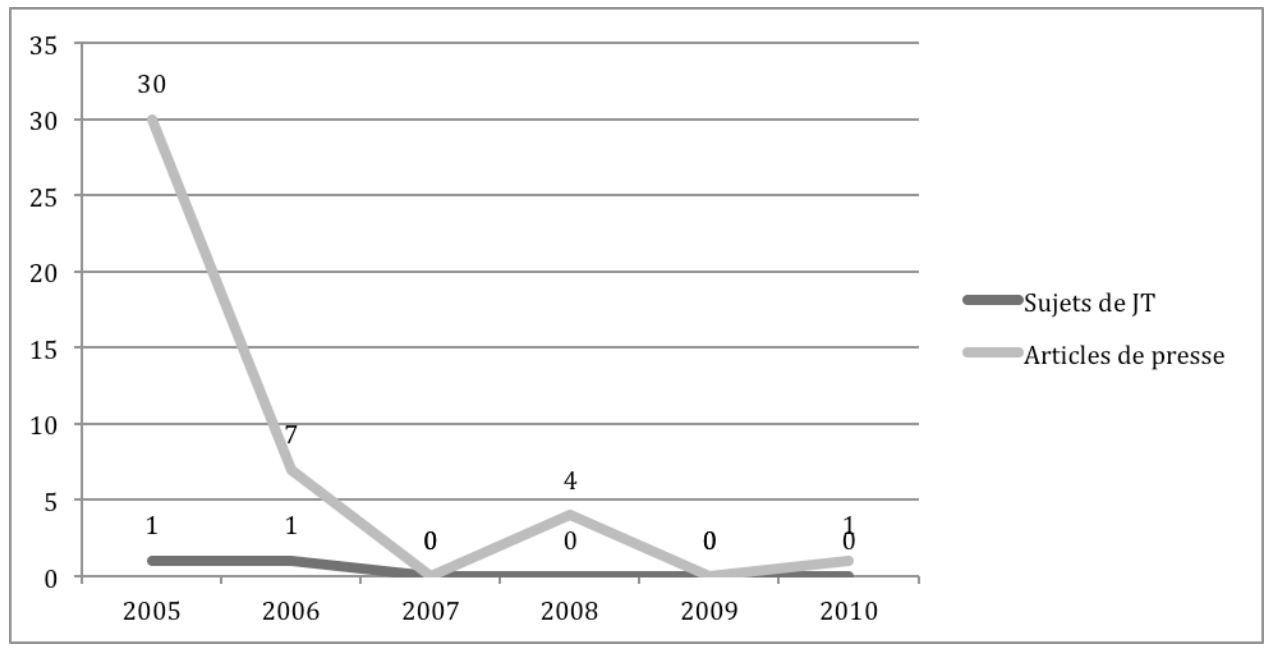

Il a également été couvert par intermittence dans la presse, et de façon inégale selon les journaux. Le Figaro et Libération ont par exemple publié respectivement 5 et 12 articles en cinq ans. Le MIR a principalement été médiatisé dans le Monde (25 articles - dont plusieurs positionnés en Une), mais cette valorisation médiatique effectuée par un seul journal a été insuffisante et trop sporadique pour que le collectif focalise réellement l'attention publique. Plusieurs actions collectives qui auraient pu bénéficier d'une (plus grande) couverture par les journalistes ont pourtant été mises en place de mai 2005 à décembre 2010, comme les différentes Marches des Indigènes (qui ont lieu tous les 8 mai pour commémorer les massacres de Sétif et de Guelma) ou l'Agora du MIR en juin 2005 (organisée pour débattre des actions à mettre en place et des revendications à porter dans le futur). En fait, le MIR a surtout été rendu visible lors de sa première année d'existence, à la suite de la diffusion de son appel et à l'occasion de la première Marche des Indigènes.

Ce peu de visibilité médiatique est significatif d'un déni de reconnaissance sociale ${ }^{3}$ qui s'est aussi matérialisé dans la représentation négative des membres du collectif ${ }^{4}$. Pour être reconnaissable dans l'espace des mobilisations collectives et interpeller l'opinion sur la cause défendue, tout groupe protestataire se doit d'être doté d'une identité publique 5 . Or, alors que le MIR s'est présenté comme étant le représentant des minorités ethnoraciales, il a été catégorisé, dans les médias, comme étant un mouvement groupusculaire, communautariste, sexiste et raciste. Les militants n'ont pas été désignés et décrits comme ils s'étaient euxmêmes auto-identifiés dans leur appel, mais ont été représentés comme incarnant une minorité dangereuse parmi les minorités ethnoraciales.

L'étude des termes utilisés pour désigner et définir les membres du MIR a mis en évidence qu'ils ont été «marqués ${ }^{6}$ par la race, mais pas par les syntagmes habituellement utilisés pour catégoriser les «non-blancs» dans les médias tels que "personnes issues de l'immigration» ou «minorités visibles ». Ce sont les multiples références à la religion musulmane qui ont ethnoracialisé les militants en les présentant comme ayant une pratique religieuse extrémiste et contraire aux valeurs de la nation ("musulmans », "des militants complaisants à l'égard de l'islam politique », "des fidèles de l'islamiste Tarik Ramadan »,

3 Honneth Axel (2006), La société du mépris. Vers une nouvelle Théorie critique, Paris, La Découverte.

$4 \quad$ Voirol Olivier (2005), «Les luttes pour la visibilité. Esquisse d'une problématique », in Réseaux $\mathrm{n}^{\circ}$ 129-130, p. 89-121.

5 Polletta Francesca and Jasper James M. (2001), «Collective identity and Social Movements », in Annual Review of Sociology n²7. p. 283-305.

6 Brekhus Wayne (2005), «Une sociologie de 1'"invisibilité" : réorienter notre regard », in Réseaux $\mathrm{n}^{\circ}$ 129-130, p. 243-272. 
« islamogauchistes », «les tenants d'un islam radical et politique », etc.).

Le MIR a également été dépeint comme étant composé de militants antiracistes et/ou d'extrême gauche («militants mobilisés contre les discriminations », " militants d'Attac, de la Ligue communiste révolutionnaire ou des droits de l'homme », "des trotskistes », " militants de gauche, d'extrême gauche ", etc.), ce qui a conduit à définir les initiateurs de l'appel comme n'ayant aucune autorité à produire un discours objectif et critique sur la (post)colonisation. Plusieurs tribunes libres rédigées par des historiens ont effectivement mis en avant l'illégitimité du MIR à émettre une position sur les conséquences contemporaines de l'Empire, à l'image d'un article du Monde ${ }^{7}$ où l'appel a été décrit comme « opportun mais pas toujours rigoureux » car, pour les auteurs de la tribune, même si «l'affirmation selon laquelle le colonialisme n'existe plus mérite à coup sûr une démonstration ", celle-ci doit uniquement venir d'universitaires, "partisans de la "complexité" ». Cette caractérisation militante s'est d'ailleurs confondue avec la religiosité de leur identité publique, identité co-construite uniquement par les journalistes et les acteurs sociaux dont le discours a été rapporté dans les médias, vu que les membres du MIR n'ont pratiquement pas eu accès à la parole publique. En étant décrits comme ayant participé à la mobilisation contre l'interdiction du port du voile à l'école en 2003 et 2004 ("une poignée d'associatifs qui s'étaient découverts dans le combat contre la loi interdisant le voile à l'école », " ennemis de la loi sur le voile », etc.), ils ont été qualifiés d' " islamogauchistes » hostiles aux valeurs de la République, ce qui a conduit à les altériser encore plus fortement attendu que, dans les imaginaires médiatiques, le port du hijab est un symbole de la soumission des femmes.

Tous les journaux étudiés ont associé le MIR à l'islamisme et à l'extrême gauche. Libération a décrit les militants comme appartenant à «des associations islamistes, soutenues par une certaine gauche ${ }^{8}$ " et le Figaro comme étant "une poignée d'associatifs qui s'étaient découverts dans le combat contre la loi interdisant le voile à l'école ${ }^{9}$ " avant de préciser qu' " autour de ce noyau se sont agrégés des militants proches du député maire PC de SaintDenis Patrick Braouzec, des fidèles de l'islamiste Tarik Ramadan, des membres d'Attac, des trotskistes ». Le Monde a quant à lui défini les auteurs de l'appel comme se situant "essentiellement au sein des courants qui avaient mené la lutte contre la loi interdisant les signes religieux à l'école. Laïcs et proches des altermondialistes comme le collectif "Les mots sont importants", musulmans comme le site oumma.com, qui diffuse le texte, ou le Collectif des musulmans de France, proche du prédicateur suisse Tariq Ramadan ${ }^{10}$ ". Par la construction d'un tel ethos négatif dans les médias d'information généraliste, les membres du mouvement (et leurs revendications) ont été socialement délégitimés et disqualifiés.

Les universitaires qui ont signé l'appel - et qui auraient pu apporter du crédit à la cause par leur statut social d'expert — n'ont pratiquement jamais été associés au MIR à l'intérieur des articles. L'identité socio-discursive des militants a plutôt été contaminée et entachée par celles de Tariq Ramadan et de Dieudonné, deux acteurs sociaux controversés dont l'antisémitisme constitue le thème de nombreux articles et sujets de JT depuis le début des années 2000. Même si les membres du MIR ne sont pas liés à ces deux individus, ils ont été décrits comme étant proches de Ramadan et rendus visible, pour la première fois, à l'intérieur d'articles traitant du dernier propos antisémite de Dieudonné, celui-ci ayant qualifié de « pornographie mémorielle » la commémoration de la Shoah en février 2005.

\section{Une thèse portée par le mouvement contestée dans les médias}

\footnotetext{
Le Monde (18/10/2005), « Aux futurs historiens du colonialisme », p. 14.

Libération (02/03/2005), «"Moi, fille d'immigrés, pour l'égalité et la laïcité" », p. 32.

Le Figaro (25/02/2005), « Les "indigènes" s'en prennent à la "France coloniale" », p. 9.

Le Monde (22/02/2005), « Des "enfants de colonisés" revendiquent leur histoire », p. 8.
} 
La médiatisation du mouvement s'est centrée sur le problème public du continuum colonial sans que les membres du collectif aient pu participer à sa configuration dans l'arène des médias. La thèse défendue par le mouvement — les indigènes des colonies et les minorités ethnoraciales partagent le même statut de sous-citoyen - a été commentée, discutée et contre-argumentée. Des universitaires, des responsables d'associations antiracistes et/ou représentant les minorités ethnoraciales (Ni putes ni soumises, SOS-Racisme, la Ligue des droits de l'Homme, etc.), des écrivains présentés comme étant "non-blancs » ainsi que des journalistes prestigieux sont intervenus dans la presse pour prendre position dans des articles d'un genre à la visée persuasive (tribunes libres, critiques ou encore articles d'analyse). Seul le Monde a publié une tribune libre rédigée par le MIR en réponse aux réactions dépréciatives qui ont suivi la diffusion de l'appel ${ }^{11}$.

Celui-ci a en fait été accusé de caricaturer la réalité sociale et de faire l'impasse sur plusieurs éléments historiques. Libération a par exemple relayé la prise de distance de certaines associations antiracistes vis-à-vis du MIR ayant jugé "les accusations portées contre la France "excessives" ou "trop simplistes"12" et même si les historiens Nicolas Bancel et Pascal Blanchard ont été les seuls acteurs sociaux à soutenir la démarche du collectif, ils ont mis en avant dans le Monde " ${ }^{13}$ [l]es raccourcis, [l]es excès et [l]es erreurs historiques » que comportaient l'appel. En dehors de cette critique, le principal argument opposé à la thèse du continuum colonial a été juridique : la correspondance entre l'Empire et la société actuelle est fausse car, en vivant sous le régime de l'indigénat, les colonisés ne jouissaient pas des mêmes droits que les métropolitains et colons, alors qu'aujourd'hui, les minorités ethnoraciales ont les mêmes droits que n'importe quel citoyen français. Cet argument a été par exemple mis en avant par Gérard Noiriel dans le Monde : "La notion d"'indigène" est une réminiscence de l'époque coloniale, où l'inégalité était juridique; elle n'indique rien quant à la position sociale 14 " et par Patrick Weil dans le Figaro: «Dire que les immigrés d'aujourd'hui sont les colonisés d'hier est absurde sur le plan historique et factuel. Contrairement aux musulmans d'Algérie pendant la colonisation, nos compatriotes de cette confession ont aujourd'hui une pleine nationalité, les mêmes droits civils que tous les Français et la liberté de conscience ${ }^{15}$. » La présidente de Ni putes ni soumises de l'époque, Fadela Amara, a usé du même type d'argument dans Libération en comparant sa situation à celle de son père : "mon père, lui, a été un vrai indigène, et sa situation n'avait rien à voir avec la mienne. Moi, je peux protester, je peux agir, je peux voter ${ }^{16}$. "

Des participants au débat public ont aussi mis en avant l'existence de «non-blancs » victimes d'inégalités qui ne sont pas originaires de pays anciennement colonisés par la France et ce pour réfuter la corrélation entre colonisation et discriminations. Jean Daniel, fondateur et directeur du Nouvel Observateur, a énoncé dans le Monde «que les Pakistanais et les Turcs, dont les ancêtres n'ont jamais été colonisés par la France, ne sont pas mieux traités que les Maghrébins ou les Africains ${ }^{17} »$. Gérard Noiriel a quant à lui désigné les gens du voyage comme étant les plus vulnérables au racisme : "Et puis ceux qui souffrent le plus aujourd'hui, ce sont les tsiganes. Alors quoi? On va dire que ce sont des "indigènes", eux aussi ? Ça ne marche pas ${ }^{18}$. » D'autres acteurs sociaux ont avancé que les discriminations étaient avant tout liées à la classe sociale et non pas à la race, comme l'écrivaine Leïla Sebbar qui a affirmé

\footnotetext{
Le Monde (17/03/2005), « Nous, "indigènes de la République" », p. 1.

Libération (07/05/2005), « Sétif revient hanter la France coloniale », p. 4.

Le Monde (17/03/2005), « "Comment en finir avec la fracture coloniale" », p.15.

Le Monde (des livres) (13/04/2007), « Démocratiser la parole », p. 12.

Le Figaro (25/02/2005), «"Dire que les immigrés sont les colonisés d'hier est absurde" », p. 9.

Le Monde (20/10/2006), « Fadela Amara. L'enfance d'une insoumise », p. 17.

Le Monde (27/03/2005), «"L'anticolonialisme comme alibi" », p. 11.

Le Monde (des livres) (13/04/2007), op. cit., p. 12.
} 
dans Libération, que, selon elle, les discriminations «s'expriment surtout contre les enfants des classes pauvres ${ }^{19} "$.

Les inégalités subies par les minorités ethnoraciales n'ont pas été discutées dans les journaux, qu'ils soient socialement identifiés comme étant à gauche ou à droite. Les discriminations directes, discriminations visibles et prouvables qui comportent une dimension individuelle et intentionnelle, ont été unanimement dénoncées et condamnées. Un journaliste de Libération a par exemple énoncé que "sur la réalité du racisme dont les Arabomusulmans sont l'objet, pas de contestation ${ }^{20} »$. Or, la cause et les revendications formulées par le MIR ne portaient pas uniquement sur ce type de discriminations : le mouvement a surtout dénoncé les discriminations systémiques qui sont significatives de rapports asymétriques entre les groupes sociaux. Il a donc été accusé d'avoir une lecture ethnoracialisée — et donc raciste — du monde social, car celle-ci s'oppose à l'universalisme républicain ${ }^{21}$.

En France, prendre en compte la race des individus et des groupes est synonyme de divisions entre les membres de la nation et reviendrait à créer du racisme. Le MIR a ainsi été décrit comme étant le véritable inégalitaire : Jean Daniel a accusé le MIR de creuser «le fossé qui sépare les Français d'origines différentes" et de "provoquer et exaspérer les antagonismes ethniques ${ }^{22}$ " et d'autres acteurs sociaux ont reproché au mouvement de victimiser les minorités ethnoraciales, c'est-à-dire de les catégoriser - en tant que groupe en fonction d'un attribut catégoriel en les définissant comme étant fondamentalement victimes d'inégalités. Un journaliste du Monde a déclaré qu' "il ne faut pas laisser faire ceux qui tentent d'attirer les jeunes issus de l'immigration [le MIR] [...] dans une identité d'éternelles victimes. Leur donner à penser que les discriminations dont ils souffrent ne seraient que le décalque de l'ancien statut des indigènes de l'Empire reviendrait à accepter l'ouverture, dans la société française, d'une inquiétante fracture coloniale ${ }^{23} »$. Ces accusations ont aussi été formulées par le président de SOS-Racisme de l'époque, Dominique Sopo: "Dominique Sopo donne l'alarme et désigne les incendiaires : "les exotiques" [le MIR], à savoir cette nébuleuse de militants qui, sous couvert d'antiracisme, s'emploieraient à répandre un extrémisme communautariste résolument hostile aux valeurs universalistes ; ceux-là enfermeraient les populations issues de l'immigration dans une posture purement victimaire, à la fois infantilisante et démobilisatrice, les réduisant "au statut d'indigènes passifs, incapables d'écrire leur histoire et leur vie" ${ }^{24}$."

Alors que les membres du mouvement ont été dépeints comme représentant une menace pour la nation, la République (mise en cause par le collectif) a été décrite dans la presse comme constituant la matrice de l'égalité entre tous les citoyens - et ce malgré son passé colonial. Le président de la Ligue des Droits de l'Homme (LDH) a affirmé que même si la France a été un Empire, c'est avant tout le pays des valeurs égalitaires et antiracistes : «on ne peut pas faire comme si l'histoire de l'Occident se réduisait au colonialisme. Les signataires du texte veulent ignorer que l'Occident a été le lieu d'élaboration de la pensée universaliste qui marque une rupture historique ${ }^{25}$. " Jean Daniel a souligné que la nation républicaine transcende la colonisation et les politiques qui l'ont mise en œuvre, car ses membres se sont toujours battus contre les inégalités et l'impérialisme ${ }^{26}$. Leïla Sebbar a quant

19 Libération (24/02/2005), «"Dieudonné se trompe de cible" », p. 33.

20 Libération (09/05/2005), «Un statut d'infériorité ressenti ou réel ? », p. 17.

21 Sur le rapport entre race et République, voir De Rudder Véronique, Poiret Christian et Vourc'h François (2000), L'inégalité raciste. L'universalité républicaine à l'épreuve, Paris, PUF.

$22 \quad$ Le Monde (27/03/2005), op. cit., p. 11.

23 Le Monde (21/01/2006), « Les blessures de la colonisation », p. 1.

24 Le Monde (25/11/2005), «L'antiracisme perverti des "exotiques" et des "obscurantistes" », p.7.

25 Le Monde (22/02/2005), « L'humoriste "tente d'instaurer une hiérarchie entre les victimes" », p. 8

26 Le Monde (27/03/2005), op. cit., p. 11 
à elle affirmé que la République protégeait ses membres des discriminations, les inégalités étant perpétuées, selon elle, en dehors des institutions de l'État: «ce n'est pas la République qu'il faut accuser [...] Les grands corps de l'État ne sont pas contaminés par le racisme contre les Noirs et les Arabes. C'est plutôt dans les secteurs incontrôlés de la société que $s^{\prime}$ expriment les discriminations ${ }^{27}{ }^{\prime \prime}$.

La République ayant été mise hors de cause, d'autres phénomènes expliquant les inégalités ont été exposées dans la presse. Pour le président de la LDH, c'est l'échec scolaire qui en est à l'origine: "Qui peut croire qu'il suffira d'introduire l'histoire coloniale ou l'histoire de l'immigration, dans les programmes de l'éducation nationale, pour régler la question de l'échec scolaire qui commande tout ? ${ }^{28} "$ et pour l'historien Gilbert Meynier, il s'agit du contexte économique : "pour reprendre le texte des "Indigènes de la République", à l'évidence, les plaies dont ils saignent sont de moins en moins celles qui sont infligées par le vieux colonialisme [...] mais bel et bien celles provoquées par la sauvagerie et la dureté économiques d'aujourd'hui ${ }^{29} »$. Alors que les membres du MIR se définissent comme antiracistes et mettent en cause la République dans leur appel, les rôles s'inversent dans la couverture médiatique du mouvement. Celui-ci est en effet présenté comme un collectif raciste contre lequel s'oppose une nation fondamentalement égalitaire.

L'étude de la couverture médiatique du MIR rend compte des possibles conséquences corrélées au port de revendications mettant en avant l'existence des rapports asymétriques de race : mettre en cause la responsabilité de la nation dans la production des inégalités, c'est énoncer un discours indicible dans la sphère publique française et s'exposer à un processus d'altérisation et de disqualification sociale. La médiatisation du mouvement est significative de la quasi-impossibilité qu'ont les individus et les groupes sociaux - qu'ils soient identifiés comme «blancs» ou «non-blancs» — à dénoncer — voire même à aborder — la problématique des rapports sociaux de race dans les médias d'information généraliste. Le débat public se structure généralement autour de la manifestation de discriminations directes perpétrées par un ou des individus « ouvertement » racistes (employeur malhonnête, électeurs du Front national, policiers faisant du contrôle au faciès...). Les discriminations systémiques ou institutionnelles font, quant à elles, rarement l'objet d'un sujet dans la presse ou à la télévision, notamment parce qu'en parler pourrait, d'une part, caractériser des groupes sociaux à l'intérieur d'une République qui refuse de prendre en compte leurs spécificités au nom de l'égalité (c'est pour cela que les inégalités sont reliées, du point de vue de la construction du sens, aux relations entre des individus et non entre les groupes), et, d'autre part, pourrait conduire à mettre en cause la participation (inconsciente) des membres « blancs » de la nation dans la production des formes systémiques de racisme. La définition de la francité risquerait alors d'être ébranlée attendu que les citoyens français sont caractérisés comme étant intrinsèquement égalitaires. 